\title{
The influence of diabetes enhanced inflammation on cell apoptosis and periodontitis
}

\author{
Tie-Lou Chen ${ }^{1 *}$, Er-Li Xu ${ }^{2 *}$, Hui-Jie Lu ${ }^{3}$, Heng Xu ${ }^{1}$, Shi-Feng Wang ${ }^{4}$, Hai-Jun Zhao ${ }^{1}$ Yu-Ming Liu ${ }^{4}$ \\ ${ }^{1}$ Department of Periodontol, Diagnosis \& Treatment Center of Stomatological Diseases of CPLA, Hospital 411 of CPLA, Shanghai, \\ China \\ ${ }^{2}$ Department of Endocrinology, Hospital 411 of CPLA, Shanghai, China \\ ${ }^{3}$ Department of Psychology, Aerospace Engineering Medical College, Fourth Military Medical University, Xi'an, China \\ ${ }^{4}$ Naval Medical Research Institute, Shanghai, China. \\ Email: *chentielou2010@sina.com,xbctl@sh163.net, erlixu@gmail.com
}

Received 16 August 2012; revised 21 September 2012; accepted 29 September 2012

\begin{abstract}
Aim: Diabetes mellitus is a metabolic disorder leading to hyperglycemia and exhibiting altered fat and protein metabolism. Diabetes altered cellular microenvironment caused myriad untoward effects. Periodontitis is chronic inflammatory disease. Diabetes and periodontitis had higher prevalence in populations. The objective studied the relationship between diabetes and periodontitis associated with cell apoptosis and the influence of diabetes enhanced inflammation on apoptosis and periodontitis. Methods: This paper studied and analyzed the papers which published in the worldwide associated with the influence of diabetes enhanced inflammation on cell apoptosis and periodontitis, and reviewed the probably mechanism associated with apoptosis. Results: Diabetes induced hyperglycemia enhanced inflammation related to cell apoptosis. Periodontitis had a higher morbidity on diabetes patients. Periodontal intervention may be benefit to controlling the diabetes. The bidirectional efficiency happened between diabetes and periodontitis. Anti-apoptotic and anti-inflammation option can improve the therapeutic effects on diabetes and periodontitis. The finding included following several aspects. 1) Advanced glycation end products enhanced inflammatory response; 2) Hyperglycemia induced cell apoptosis; 3) inflammatory cytokines caused cell apoptosis; 4) Mutuality between cell apoptosis and periodontitis; 5) Diabetes induce periodontitis and bone loss; 6) Periodontitis induced insulin resistance. 7) TNF $\alpha$ induce prostaglandins elicited cell apoptosis; 8) periodontal therapies had effects on diabetes. Conclusion: Diabetes can enhance inflamemation leading to apoptosis and periodontitis. Effective periodontal therapy and control glucose may produce better effects on diabetes or periodontitis.
\end{abstract}

*Corresponding author.
Further research required to investigate the bidirectional mechanism between diabetes and periodontitis.

Keywords: Influence; Diabetes Mellitus; Inflammation; Cell Apoptosis; Periodontitis

\section{INTRODUCTION}

Diabetes mellitus is a metabolic disorder characterized by insulin insufficiency or resistance resulting in hyperglycemia. Diabetes is associated with an increased morbidity and severe periodontitis. Inflammation is a pivotal element in the pathogenesis of diabetes. Periodontitis is chronic inflammatory disease which represented gingivitis, alveolar bone absorbed and periodontal attachment loss and even tooth loose. The interrelation between diabetes mellitus and periodontitis has been intensively studied more than 50 years. Periodontal infection can seriously impair metabolic control of diabetic and periodontal therapy has a beneficial effect on diabetes. Conversely, severe diabetes can influence the periodontal health.

About 6\% people affected diabetes worldwide in 2007 and the ratio will increase to $7.3 \%$ by 2025 . Type 1 diabetes mellitus (T1DM) caused by cellular mediated autoimmune destruction of pancreatic islet beta cells leading to loss of insulin production and started in children [1]. Type 2 diabetes mellitus (T2DM) caused by the resistance to insulin combined with unable to produce sufficient insulin, and accounted for $90 \%$ - 95\% of all diabetes and sickened after 45 years old linked to obesity [2]. The metabolic dysfunctions alter the cellular microenvironment resulting in a long-range countless effects named as "diabetic complications" including atherosclerosis and periodontitis. The inflammatory cells include macrophages, lymphocytes, neutrophils, eosinophils and dendrite cells (DC). Pro-inflammatory cyto- 
kines including tumor necrosis factor (TNF)- $\alpha$, interleukin (IL)- $1 \beta$ and IL-6 are elevated in patients with diabetes. This paper investigates the relationship between periodontitis and diabetes mellitus, with a focus on the influence of diabetes increased inflammation on cell apoptosis and periodontitis.

\section{AGES ENHANCED INFLAMMATORY RESPONSE}

\section{AGEs Induced ROS Leading to Oxidative Stress}

Advanced glycation end products (AGEs) contribute to reactive oxygen species (ROS) generation leading to oxidative stress and cell death. AGEs in diabetes patients increased inflammation via up-regulation of TNF $\alpha$ and $\mathrm{IL}-1 \beta$ in monocytes and macrophages. Alikhani M investtigated AGEs inducing apoptosis in cultures of osteoblast cells [3]. The cell apoptotic mediated through AGE receptor (RAGE) and increased in p38 and c-Jun N-terminal kinase (JNK) activity, caspase-8 and caspase-3 activation, and enhanced by AGEs in differentiated osteoblast. A study showed that AGEs reduced osseous healing and delayed bone regeneration in T1DM [4]. Methylglyoxal (MG) produced by glycolytic induced osteoblast cell death, and the elevated MG in T1DM patients may impaired bone regeneration. Apoptosis mechanism of osteoblasts induced by MG involved oxidative stress, JNK activation, mitochondrial membrane changed, cytochrome $\mathrm{C}$ released, increased $\mathrm{Bax} / \mathrm{Bcl}-2$ ratio, and activation of caspase-9, caspase-3 and p21-activated protein kinase 2 (PAK-2). Mice lacking Forkhead Box O1 (FoxO1) in osteoblasts represented $\beta$-cell proliferation, insulin secretion and insulin sensitivity increased. Osteocalcin facilitated bone mineralization which associated with elevated fasting serum glucose in T2DM patients [5].

Inflammatory cytokines enhance vascular permeability and leukocyte adhesion to endothelium, which in turn change vasoregulatory responses and facilitate thrombus formation by inducing procoagulant activity and inhibiting anticoagulant. Chen TL represented that periodontitis and gingivitis had the higher contents of thromboxane $\mathrm{B}_{2}$ and 6-keto-prostaglandin $F_{1} \alpha$ in gingival and the increased levels are associated with the increased inflamematory cells and vessel endothelial cells, which can regulate the vasoconstriction and vasorelaxation and inflammatory cytokine secreted and released directly or indirectly leading to destruction of periodontal tissue [6,7]. Serum C-reactive protein (CRP), IL-6, IL-1, TNF $\alpha$ and fibrinogen increased in diabetes [8]. Nuclear factor kappa B (NF- $\kappa \mathrm{B})$ activated by TNF $\alpha$ and IL-1 next to hyperglycemia, AGEs and insulin to translocation from the cytoplasm to the nucleus to activate gene transcription. NF- $\kappa \mathrm{B}$ regulate vascular inflammatory response which increased adhesion of monocytes, neutrophils, and macrophages leading to cell impairment.

\section{HYPERGLYCEMIA INDUCE CELL APOPTOSIS}

Hyperglycemia leading to cell apoptosis includes increased oxidative stress and intracellular $\mathrm{Ca}^{2+}$, mitochondrial dysfunction; intracellular fatty acid metabolism changed, activation of mitogen, and impaired phosphorylation of protein kinase Akt [9]. One study with human umbilical vein endothelial cells (HUVECs) demonstrated that elevated glucose inducing apoptosis and down-regulating vascular endothelial growth factor (VEGF) in HUVECs by inhibiting p42/44 MAP kinase activation. High glucose increased Bax protein and elevated the $\mathrm{Bax} / \mathrm{Bcl}-2$ ratio can activate procaspase 3 into active caspase-3 and trigger HUVECs apoptosis. Apoptosis prevented through inhibition of increased ROS generation and activation of the mitochondria apoptosis when VEGF added to the HUVECs exposed to high glucose. VEGF decreased Bax expression without influencing $\mathrm{Bcl}-2$ attenuated caspase 3 activity and lessen $\mathrm{H}_{2} \mathrm{O}_{2}$ production high glucose stimulation at 48 hours and inhibited ROS/NF- $\kappa \mathrm{B} / \mathrm{JNK} /$ Caspase-3 pathway in HUVECs [10]. One study with aortic endothelial cells on high glucose showed increased Bax/Bcl-2 ratio followed by an increase in caspase-3 activity and cell death. High glucose can trigger HUVECs apoptosis via ROS through activating c-Jun NH2-Terminal Kinase/stress activated protein kinase (JNK/SAPK) [11]. HUVECs treated with high glucose for 24 hours indicated a causal relation of changing intracellular fatty acid and apoptosis in hyperglycemia. Hyperglycemia can regulate cyclooxygenase 2 (COX-2) expressions and increase of prostaglandin $E_{2}$ $\left(\mathrm{PGE}_{2}\right)$ production and subsequently a caspase-3 activation and foster the apoptosis of HUVECs. Inhibition of COX-2 inhibitor NS398 decreased $\mathrm{PGE}_{2}$ production, caspase-3 activity and apoptosis in HUVECs which can prevent high glucose. Hyperglycemia can trigger NF- $\kappa \mathrm{B}$ activation and COX-2 expression, induced COX-2 mediated $\mathrm{PGE}_{2}$ production and apoptosis in HUVECs exposed to hyperglycemia [12]. Accumulated number of cellular $\mathrm{Ca}^{2+}$ caused more mitochondrial $\mathrm{Ca}^{2+}$ uptake and enhanced mitochondrial permeability transition and exerted a key role in cell apoptosis.

The metabolic associated with an increase in caspase- 3 activity and an impaired of insulin to activate Akt. Recently, a study with human pancreatic micro vascular endothelial cells (MECs) showed that sustained hyperglycemia progressively affected cellular survival and proliferation leading to the MECs apoptosis increased. Another study on MECs under sustained hyperglycemia represented a progressively reduced phosphorylation of 
Akt and an interference with Akt activation. Hyperglycemia down-regulated tyrosine phosphorylated form the transmembrane protein. The results showed that hyperglycemia induced apoptosis of islet endothelium involved the nephrin mediated signaling cascade. Studying with islet MECs detected the increased IL- $\beta$ can analyze Fas expression and Fas-mediated apoptosis [9].

\section{INFLAMMATORY CYTOKINES INDUCE CELL APOPTOSIS}

Apoptosis is a controlled and regulated process and plays an active role in cell death or cell suicide. Necrosis is an uncontrolled process of cell lyses leading to inflammation and destruction of tissue, which can cause serious health problems. Apoptosis act as a protection effect on eliminating old, useless, and damaged cells during human life. Apoptosis and cell proliferation are in balance in healthy organisms, but an imbalance station in diseases prevents them from undergoing apoptosis. CollinOsdoby P [13] found that nitric oxide (NO) can induce osteoblast apoptosis, and enhanced NO leading to oxidative stress and osteoblast death. TNF $\alpha, \mathrm{IL}-1 \beta$ and interferon gamma (IFN $\gamma$ ) caused activation of the inducible NOS (iNOS) in bone and enlarged NO potentiates bone loss. NO can inhibit endothelial cells apoptosis, and endothelial NO syntheses (eNOS) expressed in bone and iNOS expressed only in response to inflammatory stimuli. The eNOS isoform play an important role in regulating osteoblast activity and bone formation and iNOS required for bone repair in mice. Elevated serum TNF $\alpha$ levels showed direct correlation with vascular iNOS expression and a possible link between inflammation and reduced bone mass in T2DM [14]. Increased inflammatory cytokine $\mathrm{PGE}_{2}$ in gingiva and gingival crevicular fluid with periodontal diseases associated with increased macrophages and plasmacytes in gingiva with periodontitis and gingivitis leading to tissue impaired [15].

Cell death inducing ligands include Fas ligand, TNF $\alpha$ and TRAIL. Apoptotic signals transmitted and a caspase cascade activated would induce cell apoptosis when binding to death receptor to amplify the apoptosis signal. A change brings out the presence of a death domain allowing the recruitment of different apoptotic proteins to the receptor. The sensitivity of cells on apoptosis depends on the balance of pro- and anti-apoptotic bcl-2 proteins. Bcl-2 and bcl-XL are anti-apoptotic, but Bad, Bax and Bid are pro-apoptotic proteins [10]. The interaction between pro- and anti-apoptosis proteins leaded to the formation of permeability transition pores (PTP) in the mitochondrial membranes. The mitochondria contain pro-apoptosis proteins (cytochrome C) and released through these pores leading to the formation of the apoptosome and activation of the caspase cascade [16].
Once cytochrome $\mathrm{C}$ released into the cytosol, apoptotic peptidase activating factor-1 (APAF-1) leaded to the recruitment of procaspase 9 into apoptosome. The antiapoptosis effects mediated through nitrosylation and inactivation of caspase 1, 3 and 8 and activating p53, and anti-apoptotic proteins Bcl-2 and Bcl-XL. Caspase activity suppressed through activation of cGMP signaling and endothelial cells apoptosis is critical in diabetes [17]. Apoptosis can be induced by extrinsic signals binding to cell surface receptors called death receptors and by intrinsic signals following cellular stress and resulted from oxidative stress through free radicals. The deficiency of immune system in the no obese diabetic (NOD) mouse showed the predisposition of NOD to T1DM [18].

T1DM in NOD mice detected according to infiltration of pancreatic islets with macrophages, $\mathrm{B}$ cells, $\mathrm{CD} 4^{+}$and CD8+ T cells. Insulitis leads to the preferential amplification of auto reactive CD8+ T cells with high affinity $\mathrm{T}$ cell receptors (TCR), and high affinity pre-cytotoxic $\mathrm{T}$ lymphocytes (CTLs) differentiated into CTLs. CD8 ${ }^{+}$ CTLs started the immune response via the production of perforin. TNF- $\alpha$, IFN- $\gamma$, and IL- $1 \beta$ up-regulate Fas expression and stimulate NO and ROS production exacerbating cell death [19]. The mechanisms of apoptosis in T1DM include increased serum cell nutrients, endoplasmic reticulum (ER) stress and infiltration of immune cells. The proinflammatory cytokine IL- $1 \beta$ is one of the unifying mechanisms of $\beta$-cell death and the expression of IL- $1 \beta$ in pancreatic $\beta$-cells with T2DM Increased. A study found that the natural soluble IL-1 receptor antagonist (IL-1Ra) in diabetes can improve glycated hemoglobin levels in IL-1Ra treated than placebo patients, but the study observed only 13 weeks [20]. The apoptosis of pancreatic islets $\beta$-cell from T2DM increased, and islet macrophage infiltration of T2DM occurred before cell death.

\section{CELL APOPTOSIS AND PERIODONTITIS}

Diabetes impaired immune function and increased risk of bacterial, viral and fungal infections. A study, which had poor glycaemic control, showed lessened chemotactic activity and bactericidal activity leading to reduced ROS and lysosomal enzyme released [21]. Impairment of the inflammatory response with hyperglycemia mediated alteration in lipid and protein function can result in AGEs and methglyxol formation in cell culture and human exvivo experiment. Apoptotic lymphocytes occurred in diabetic reduced numbers of plasma lymphocytes in the patients [22]. Expanded apoptosis of lymphocytes in diabetes may elucidate the impaired immune function in poorly controlled diabetic patients. Neutrophil apoptosis is an integral component of inflammation and its resolu- 
tion particularly. The enhanced loss of fibroblasts and osteoblasts through apoptosis in diabetics could contribute to limited repair of injured tissue, and influent the wound healing of periodontal tissue [23].

Chen TL reported the molecular mechanisms of apoptosis on the onset of periodontitis and investigated the molecular control mechanism of apoptosis on periodontitis. The finding indicated the control gene of apoptosis were mainly p53, Bcl-2, c-mys. Reciprocity of Fas and Fasl is related to cell apoptosis. Two major pathways are involved in the process of apoptosis in periodontitis. One is the intrinsic pathway for apoptosis induced by mitochondria, known as the intrinsic pathway. The death receptor (Fas/FasL) has been involved in the second pathway, also known as the extrinsic pathway. The cell apoptosis is also related to lipid peroxide. Tetracycline and Vitamin $C$ has the therapeutic effect on periodontitis by inhibiting apoptosis [24].

\section{DIABETES INDUCE PERIODONTITIS AND BONE LOSS}

Anaerobes bacteria are the dominating pathological bacteria of periodontitis. Periodontal bacteria and secretion leading to inflammatory response resulted in periodontal tissue breakdown. Alveolar bone has the ability for bone remodeling and regeneration. Bacterial plaque accumulated on the tooth surface can stimulate the host response in the adjacent gingival and resulted in the destruction of periodontal tissue, and periodontal bone loss is the critical characters of periodontitis [25]. Periodontal bone loss appeared when the bone absorption exceeds new bone formation. Diabetic represented inflammatory response result of hyperglycemia. A study showed that T1DM reduced the formation of new bone and decreased bone mineral density leading to osteopenia. The impact of T1DM on bone is reflected by a significant delay in fracture healing. Both T1DM and T2DM increased the risk of periodontitis 3 to 4 times. There is reduced fracture healing or osseous repair after marrow ablation in diabetics compared with normal's [26]. Bacterial insulting can induce the apoptosis of bone-lining cells and diabetes had an intense effect on apoptosis of bone-lining cells. Bone surfaces in diabetic mice are lined by fewer cells than bone in normal and the increased apoptosis of bone-lining cells decreased the bone formation [27]. Soft tissue wounds indicated diabetic mice had increased levels of apoptosis, and diabetes influence on apoptosis of matrix-producing cells and limit the repair of injured tissue. Tuominen [28] indicated that the reduced bone mass in T1DM had a higher bone loss and a profound effect on bone remodeling than T2DM. An inflammatory stimulus in animal model of T2DM showed the inhibittion of osteoclastogenesis represented reducing new bone formation.

The mechanisms of hyperglycemia on periodontitis described as following [29]. Firstly, hyperglycemia leading to increase gingival crevicular fluid influences the microbial flora such as biofilm and accelerates the inflammatory processes in the mouth and alters the immune response of the periodontal bacteria infectious leading to the breakdown of perodontium. Secondly, hyperglycemia increases the sensitivity of bacteria to diabetes patients and alters the chemotaxis and adherence to neutrophil resulting in producing much inflammatory cytokine. Hyperglycemia increased the levels of AGEs leading to pathological biochemical processes such as glycation of protein-like collagens or lipids and nonenzymatic oxidative destruction. AGEs can influence normal protein functions directly or act by reacting with receptors indirectly on the different cell membrane. The glycated products had the potential to create molecular complexes reducing the solubility of the target proteinlike collagens and alter the functional properties of type 1 collagen and lamina. Interactions between AGEs and receptors mediated the expression of cytokines and growth factors by macrophages. Inflammatory responses induced by AGEs contribute to systemic degradation of periodontal tissue in diabetic patients. Blockade of receptors for the AGEs reduced alveolar bone loss and the effects of oxidative stress by blocking the activation of innate immunity may be used to treat periodontitis [30]. Thirdly, increased inflammatory cytokines and secretion resulted in insulin resistance and in turn caused periodontal infection stimulate immune activity cell to release a number of inflammatory cytokine TNF1 $\alpha$ and IL-1. TNF1 $\alpha$ inhibited phosphorylation of insulin receptor and lessen the sensitivity of insulin leading to insulin resistance. Diabetes mellitus are associated with altered collagen metabolism and increased bacteria pathogenic to periodontal tissue and thereby increased the severity of periodontitis. Matrix metalloproteinase involved in a number of physiological events and as the major option in collagen breakdown and periodontal tissue destruction. An increased levels of matrix metalloproteinase 8 and 9 in the gingival tissue of diabetic with periodontitis suggested that expression of matrix metalloproteinase's contributes to failure of the healing in the diabetic. Periodontal therapy could improve tissue healing in chronic periodontitis by inhibition of matrix metalloproteinase [31]. Periodontitis with diabetics enhanced susceptibility to infection due to diminished neutrophil recruitment and function and increased formation of inflammatory cytokines and delayed wound healing after bacterial inbreak. Bone loss increased because the effects of diabetes inhibited new bone formation and the apoptosis of bone lining cells increased. Enhanced expression of cytokines in vitro is capable of stimulating bone absorption in dia- 
betics. And enhanced inflammation and bone absorption may increase risk and severity of periodontitis with diabetes [32].

\section{PERIODONTITIS INDUCED INSULIN RESISTANCE}

Increased proinflammatory cytokines such as PGE $_{2}$, IL-1, and TNF $\alpha$, IL-6 are associated with diabetes and the formation of AGEs. Reduced the over expression of cytokines by preventing from AGEs can inhibit alveolar bone loss stimulated by $P$. gingivalis in diabetic mice [30]. Periodontitis is a cascade event including the increase of cytokine, activation of acute-phase protein synthesis and consequent insulin resistance producing pathogenic changes leading to T2DM. Periodontitis leading to an increase in serum TNF $\alpha$, CRP, IL- 1 and IL-6 may induce insulin resistance by interfering with glucose and lipid metabolism [33]. The increased insulin resistance ultimately caused an increase in the risk for T2DM. One study showed the direct bacterial effects on platelets and host cells induced inflammatory mediators and autoimmune response. The increase of TNF $\alpha$, CRP, IL-6, IL-1 and fibrinogen involved in atherosclerotic plaques can lead to periodontal tissue destruction [34]. Periodontitis and diabetes mellitus could contribute to similar features of inflammation with CRP and IL-6. The severity of periodontitis and the serum TNF $\alpha$ levels are closely linked with insulin resistance [35].

Host cells released IL- $1 \alpha$, IL- $1 \beta$ and TNF $\alpha$ when stimulated by bacterial pathogens leading to reactive oxygen species (ROS) in periodontitis increased. The cytokines stimulated PMNs and in turn induced proteolysis enzymes and ROS enhanced. The imbalance between production of ROS and antioxidant defense enhanced oxidative stress. The formation of superoxides mediated activation of the polyol pathway, the hexosamine pathway, protein kinase $C$ and the formation of AGEs [36]. Inflammatory responses induced by AGEs may impair connective tissue in diabetic and increased the risk of diabetic complications. Bluher et al. [37] found a significant increase serum CRP, IL-6 and IL-8, and a critical decrease in IL-10 with the impairment of glucose tolerance. The study indicated that insulin resistance associated with an exaggerated acute phase response could precede the development of T2DM. A cohort study showed that the increase in pocket depth was more associated with the development of glucose intolerance rather than past glucose tolerance status, and periodontal infectious may couple with poor metabolic control of diabetes [38]. Nelson et al. [39] represented that the extent of periodontitis associated with glycemic control and diabetics had poor glycemic control presented more severe periodontitis. A researcher bring forth that periodontitis is a risk factor for poor glycemic control and poorly controlled diabetes, and leading to complications of diabetes. Nibali et al. [40] indicated that untreated severe periodontitis may be at increased risk of diabetes and leading to increase insulin resistance and reduce glucose tolerance.

\section{TNF $\alpha$ INDUCED CELL APOPTOSIS}

\subsection{TNF $\alpha$ Induce Prostaglandins Leading to Apoptosis}

Increased serum $\mathrm{TNF} \alpha$ in the diabetic patients with periodontitis enhanced insulin resistance. TNF $\alpha$ induced liberation of arachidonate from diacylglycerol and increased prostaglandin synthesis in cultured osteoblasts. The prostaglandin 15-deoxy-delta 12, 14-prostaglandin J2 (15d-PGJ2) can induce cell apoptosis and provoke cell death in mouse osteoblastic cell cultures. Oxidative injury showed a primary event following 15d-PGJ2 therapy resulting in Akt inactivation and leading to mitochondrial injury and apoptosis [41]. Hyperglycemia can increase osteoclast survival overshadowed apoptosis in animal model of T2DM. Increased osteoclast activity is the net effect of diabetes associated factors, such as elevated levels of saturated fatty acids, low density lipoproteins, prostaglandins and AGEs can interfere with the RANK and caspase-3 pathways [42]. Increased inflamematory mediator transforming growth factor- $\beta$ can advance osteoclast survival through up-regulation of leukemia inhibitory factor and suppressor of cytokine signaling-3 expression. Osteoblasts and stromal cells of the bone create RANKL to help osteoclast survival [43]. Osteoprotegerin (OPG) mainly produced in bone and connective tissues to prevent RANK signaling and induce osteoclast apoptosis. OPG increased in diabetic patients and OPG expression and production regulated by inflammatory cytokines. IL- $1 \beta$, TNF $\alpha$ and IFN $\gamma$ increased NO production 50 to 70 folds in osteoblasts and the extra NO liberation leading to osteoclast death in vitro and showed proinflammatory signals helping to osteoclast survival [44].

\subsection{The TNF $\alpha$ Regulate Immune Response and Cell Apoptosis}

$\mathrm{TNF} \alpha$ produced by neutrophils and macrophages can induce IL-6 production and regulate the expression of CRP. CRP increased the expression of endothelial ICAM-1, VCAM-1, E-selectin, and MCP-1 and the secretion of ET1, and decreased eNOS expression and elevated the expression of angiotensin receptor type 1 in the vessel wall [45]. TNF $\alpha$ induced insulin resistance and endothelial dysfunction. A study with diabetes rats found TNF $\alpha$ induced micro vascular cell apoptosis of diabetes, 
and enhanced TNF $\alpha$ in turn increased FoxO1 mRNA levels, nuclear translocation, and DNA binding in retinas. The results showed that the FoxO1, which regulated cell death and prevented cell cycle progression, could induce cell apoptosis and micro vascular cell loss of diabetes [46]. Type II membrane protein (TRAIL) caused minimal organ toxicity and inflammation. NO released by vascular endothelial cells and reacted on diabetic vasculopathy through down-regulation of TRAIL expression. Serum CRP increased in diabetes and played a critical role in endothelial cells, vascular smooth muscle cells and macrophages [47]. T2DM represented oxidative stress and chronic inflammation caused the primed polymorph nuclear leukocytes (PMNs) released superoxide faster than control PMNs, and the apoptosis was higher in critical PMNs with diabetes [48].

\section{PERIODONTAL THERAPIES ON DIABETES}

Periodontal therapy may perfect the metabolic control diabetes via improving insulin sensitivity and lessening the peripheral TNF $\alpha$. A significant decrease of TNF $\alpha$ was related to the reduction of total HbA1c levels post periodontal therapy. Grosse et al. [49] found that effective control periodontal infection in diabetic could reduce the serum AGEs, the volume of gingival crevicular fluid and the levels of IL-1 $\beta$ and TNF $\alpha$. Sanchez ABN [50] showed that metabolic control in diabetic subjects can be improved by the reductions in HbA1c and decreased serum TNF $\alpha$ and fibrinogen at 3 months post therapy. Periodontal therapy reduced the serum CRP and HbA1c, and in turn inhibited the diabetic process and improved glycemic control in T2DM.

Non-surgical periodontal intervention combined with systemic doxycycline showed a significant improvement in periodontitis and a short-term reduction in HbA1c levels. Non-surgical therapy combination with modulation of host response can improve the balance between resolution and development in the disease healing. Doxycycline can reduce tissue destruction and stabilize the periodontium by regulating protection of the host response. The inhibition of active matrix metalloproteinase via oxidative activation regulates expression of inflammatory cytokines and stimulates fibroblast activity resulting in a reduction in osteoclastic activity and bone absorption [51]. A potential agent in treatment of insulin resistance exerts anti-inflammatory and apoptosis modulating profile [52]. Periodontal therapy may prevent the complications of diabetes and influence on mortality by altering glycemic control and reduce the levels of HbA1c. Lalla et al. [53] found that the periodontal therapy can significant suppress serum CRP, TNF $\alpha$, soluble E-selectin, and monocytes. Curcumin inhibited osteoclast survival and protected chondrocytes from apoptosis which demonstrated a strong potential in combating of inflammation, insulin resistance and chondrocyte death.

\section{CONCLUSION}

Diabetes mellitus is a metabolic disorder resulting in hyperglycemia and altered cellular microenvironment caused unwanted effects. Periodontitis is chronic inflamematory diseases. Diabetes and periodontitis had higher morbidity in the world. There are the bidirectional influence between diabetes and periodontitis associated with cell apoptosis. Anti-apoptotic and anti-inflammation option can improve the therapeutic effects on diabetes and periodontitis. AGEs enhanced inflammatory response, and hyperglycemia and inflammatory cytokines induced cell apoptosis, and diabetes induced periodontitis and bone loss, and periodontitis induced insulin resistance. Diabetes can significantly enhanced inflammation leading to apoptosis and periodontitis. Effective periodontal therapy and rational control glucose may produce better effects on diabetes or periodontitis. The mechanism on diabetes induced inflammatory and enhanced apoptosis and periodontitis will be much clear following detailed research.

\section{ACKNOWLEDGEMENTS}

This study was supported by Nanjing Command Science \& Technology Program (06MA08) and District Key Foundation of Science \& Technology Program of Shanghai (1002-04). We also wish to thank all the contributors for the substantial information that was compiled in previously published papers and reviews that were cited in the manuscript. The information was vastly helpful for preparing the manuscript.

\section{REFERENCES}

[1] Rossini, A.A., Mordes, J.P. and Like, A.A. (1985) Immunology of insulin-dependent diabetes mellitus. Annual Review of Immunology, 3, 289-320. doi:10.1146/annurev.iy.03.040185.001445

[2] Kahn, B.B. and Flier, J.S. (2000) Obesity and insulin resistance. Journal of Clinical Investigation, 106, 473481. doi:10.1172/JCI10842

[3] Alikhani, M., Alikhani, Z., Boyd, C., MacLellan, C.M., Raptis, M. and Liu, R. (2007) Advanced glycation end products stimulate osteoblast apoptosis via the MAP kinase and cytosolic apoptotic pathways. Bone, 40, 345353. doi:10.1016/j.bone.2006.09.011

[4] Santana, R.B., Xu, L., Chase, H.B., Amar, S., Graves, D.T. and Trackman, P.C. (2003) A role for advanced glycation end products in diminished bone healing in type 1 diabetes. Diabetes, 52, 1502-1510. doi:10.2337/diabetes.52.6.1502

[5] Kanazawa, I., Yamaguchi, T., Yamamoto, M., Yamauchi, M., Kurioka, S. and Yano, S. (2009) Serum osteocalcin 
level is associated with glucose metabolism and atherosclerosis parameters in type 2 diabetes mellitus. Journal of Clinical Endocrinology \& Metabolism, 94, 45-49. doi:10.1210/jc.2008-1455

[6] Chen, T.L., Zhou, Y.J. and Wu, Z.F. (1994) Distributive and quantitative analysis of thromboxane $B_{2}$ in gingival with periodontal diseases by radioimmunoassay and immunohistochemistry. West China Journal of Stomatology, 12, 241-243.

[7] Chen, T.L., Zhou, Y.J. and Wu, Z.F. (1994) Quantitative and distributive analysis of 6-keto-prostaglandin $\mathrm{F}_{1 \alpha}$ in gingival with periodontal diseases by radioimmunoassay and immunohistochemistry. Journal of Clinical Stomatology, 10, 199-202.

[8] Folsom, A.R., Rosamond, W.D. and Shahar, E. (1999) Prospective study of markers of hemostatic function with risk of ischemic stroke, the atherosclerosis risk in communities (ARIC) study investigators. Circulation, 100, 736-742. doi:10.1161/01.CIR.100.7.736

[9] Favaro, E., Miceli, I. and Bussolati, B. (2008) Hyperglycemia induces apoptosis of human pancreatic islet endothelial cells: effects of pravastatin on the Akt survival pathway. American Journal of Pathology, 173, 442-450. doi:10.2353/ajpath.2008.080238

[10] Yang, Z., Mo, X. and Gong, Q. (2008) Critical effect of VEGF in the process of endothelial cell apoptosis induced by high glucose. Apoptosis, 13, 1331-1343. doi:10.1007/s10495-008-0257-y

[11] Ho, F.M., Lin, W.W. and Chen, B.C. (2006) High glucose-induced apoptosis in human vascular endothelial cells is mediated through NF- $\kappa \mathrm{B}$ and c-Jun NH2-terminal kinase pathway and prevented by PI3K/Akt/eNOS pathway. Cellular Signalling, 18, 391-399. doi:10.1016/j.cellsig.2005.05.009

[12] Sheu, M.L., Ho, F.M. and Yang, R.S. (2005) High glucose induces human endothelial cell apoptosis through a phosphoinositide 3-kinase-regulated cyclooxygenase-2 pathway. Arteriosclerosis, Thrombosis, and Vascular Biology, 25, 539-545. doi:10.1161/01.ATV.0000155462.24263.e4

[13] Collin-Osdoby, P., Nickols, G.A. and Osdoby, P. (1995) Bone cell function, regulation and communication: A role for nitric oxide. Journal of Cellular Biochemistry, 57, 399-408. doi:10.1002/jcb.240570305

[14] Charbonneau, A. and Marette, A. (2010) Inducible nitric oxide synthase induction underlies lipid-induced hepatic insulin resistance in mice: Potential role of tyrosine nitration of insulin signaling proteins. Diabetes, 59, 861-871. doi:10.2337/db09-1238

[15] Chen, T.L., Zhou, Y.J., Wu, Z.F. and Jin, Y. (1995) Quantitative analysis of prostaglandin $\mathrm{E}_{2}$ in gingival with periodontal diseases by radioimmunoassay and immunohistochemistry. Chinese Journal of Conservative Dentistry, 5,141-143

[16] Kluck, R.M., Bossy-Wetzel, E. and Green, D.R. (1997) New-mitochondria: A primary site for Bcl-2 regulation of apoptosis, Science, 275, 1132-1136. doi:10.1126/science.275.5303.1132

[17] Davidson, S.M. and Duchen, M.R. (2007) Endothelial mitochondria: Contributing to vascular function and disease. Circulation Research, 100, 1128-1141. doi:10.1161/01.RES.0000261970.18328.1d

[18] Wicker, L.S., Todd, J.A. and Peterson, L.B. (1995) Genetic control of autoimmune diabetes in the NOD mouse. Annual Review of Immunology, 13, 179-200. doi:10.1146/annurev.iy.13.040195.001143

[19] McKenzie, M.D., Dudek, N.L. and Mariana, L. (2006) Perforin and Fas induced by IFN gamma and TNF alpha mediate beta cell death by OT-I CTL. International Immunology, 18, 837-846. doi:10.1093/intimm/dxl020

[20] Larsen, C.M., Faulenbach, M. and Vaag, A. (2007) Interleukin-1-receptor antagonist in type 2 diabetes mellitus. New England Journal of Medicine, 356, 1517-1526. doi:10.1056/NEJMoa065213

[21] Alba-Loureiro, T.C., Munhoz, C.D. and Martins, J.O. (2007) Neutrophil function and metabolism in individuals with diabetes mellitus. Brazilian Journal of Medical and Biological Research, 40, 1037-1044. doi:10.1590/S0100-879X2006005000143

[22] Otton, R., Soriano, F.G., Verlengia, R. and Curi, R. (2004) Diabetes induces apoptosis in lymphocytes. Journal of Endocrinology, 182, 145-156. doi:10.1677/joe.0.1820145

[23] Graves, D.T., Liu, R. and Alikhani, M. (2006) Diabetesenhanced inflammation and apoptosis impact on periodontal pathology. Journal of Dental Research, 85, 15-21. doi:10.1177/154405910608500103

[24] Chen, T.L. and Wu, Z.F. (2011) The molecular mechanisms of apoptosis on the onset of periodontitis. Journal of Tongji University, 32, 116-119.

[25] Williams, R. (1990) Periodontal disease. New England Journal of Medicine, 322, 373-382. doi:10.1056/NEJM199002083220606

[26] Herskind, A.M., Christensen, K., Norgaard-Andersen, K. and Andersen, J.F. (1992) Diabetes mellitus and healing of closed fractures. Diabetes \& Metabolism, 18, 63-64.

[27] Weinstein, R., Jilka, R., Parfitt, A. and Manolagas, S. (1998) Inhibition of osteoblastogenesis and promotion of apoptosis of osteoblasts and osteocytes by glucocorticoids. Potential mechanisms of their deleterious effects of bone. Journal of Clinical Investigation, 102, 274-282. doi:10.1172/JCI2799

[28] Tuominen, J., Impivaara, O., Puukka, P. and Ronnenmaa, T. (1999) Bone mineral density in patients with type 1 and type 2 diabetes. Diabetes Care, 22, 1196-1200. doi:10.2337/diacare.22.7.1196

[29] Ryan, M.E., Carnu, O. and Kamer, A. (2003) The influence of diabetes on the periodontal tissues. Journal of the American Dental Association, 1, 34S-40S.

[30] Lalla, E., Lamster, I.B., Feit, M., Huang, L., Spessot, A., Qu, W., Kislinger, T., Lu, Y., Stern, D.M. and Schmidt, A.M. (2000) Blockade of RAGE suppresses periodontitis associated alveolar bone loss in diabetic mice. Journal of Clinical Investigation, 105, 1117-1124. doi:10.1172/JCI8942

[31] Soell, M., Hassan, M., Miliauskaite, A., Haı-kel, Y. and Selimovic, D. (2007) The oral cavity of elderly patients in diabetes. Diabetes \& Metabolism, 1, S10-S18. 
doi:10.1016/S1262-3636(07)80053-X

[32] Kurtis, B., Devlioglu, H., Taner, I., Balos, K. and Tekin, I. (1999) IL-6 levels in gingival crecicular fluid (GCF) from patients with non-insulin dependent diabetes mellitus (NIDDM), adult periodontitis and healthy subjects. Journal of Oral Science, 41, 163-167.

doi:10.2334/josnusd.41.163

[33] Grossi, S.G. and Genco, R.J. (1998) Periodontal disease and diabetes mellitus: A two-way relationship. Annals of Periodontology, 3, 51-61. doi:10.1902/annals.1998.3.1.51

[34] Chen, T.L., Wang, S.F., Liu, G.Q., Zhang, X.H., Tang, D.H. and Wu, Z.F. (2012) Influence of periodontitis and nonsurgical periodontal intervention on atherosclerosis diseases. Advances in Bioscience and Biotechnology, 3, 531-537. doi:10.4236/abb.2012.324070

[35] Mealey, B.L. and Rose, L.F. (2008) Diabetes mellitus and inflammatory periodontal disease. Compendium, 29, 403413.

[36] Ritchie, C.S. (2009) Mechanistic links between type 2 diabetes and periodontitis. Journal of Dentistry, 37, 567584. doi:10.1016/j.jdent.2009.05.015

[37] Bluher, M., Fasshauer, M., Tonjes, A., Kratzsch, J., Schon, M.R. and Paschke, R. (2005) Association of interleukin-6, C-reactive protein, interleukin-10 and adiponectin plasma concentrations with measures of obesity, insulin sensitivity and glucose metabolism. Experimental and Clinical Endocrinology \& Diabete, 113, 534-537. doi:10.1055/s-2005-872851

[38] Southerland, J.H., Taylor, G.W. and Offenbacher, S. (2005) Diabetes and periodontal infection: Making the connection. Clinical Diabetes, 23, 171-178. doi:10.2337/diaclin.23.4.171

[39] Nelson, R.G., Shlossman, M., Budding, L.M., Pettitt, D.J., Saad, M.F., Genco, R.J. and Knowler, W.C. (1990) Periodontal disease and NIDDM in Pima Indians. Diabetes Care, 13, 836-844. doi:10.2337/diacare.13.8.836

[40] Nibali, L.D., Aiuto, F., Griffiths, G., Patel, K., Suvan, J. and Tonetti, M.S. (2007) Severe periodontitis is associated with systemic inflammation and a dysmetabolic status: A case-control study. Journal of Clinical Periodontology, 34, 931-937. doi:10.1111/j.1600-051X.2007.01133.X

[41] Shin, S.W., Seo, C.Y., Han, H., Han, J.Y., Jeong, J.S. and Kwak, J.Y. (2009) 15d-PGJ2 induces apoptosis by reactive oxygen species-mediated inactivation of Akt in leukemia and colorectal cancer cells and shows in vivo antitumor activity. Clinical Cancer Research, 15, 5414-5425. doi:10.1158/1078-0432.CCR-08-3101

[42] Cornish, J., MacGibbon, A., Lin, J.M., Watson, M., Callon, K.E. and Tong, P.C. (2008) Modulation of osteoclastogenesis by fatty acids. Endocrinology, 149, 56885695. doi:10.1210/en.2008-0111

[43] Perez-Sayans, M., Somoza-Martin, J.M., Barros-Angueira, F., Rey, J.M. and Garcia-Garcia, A. (2010) RANK/ RANKL/OPG role in distraction osteogenesis. Oral Surgery, Oral Medicine, Oral Pathology, Oral Radiology, and Endodontology, 109, 679-686. doi:10.1016/j.tripleo.2009.10.042

[44] Van’t, Hof, R.J. and Ralston, S.H. (1997) Cytokine-induced nitric oxide inhibits bone resorption by inducing apoptosis of osteoclast progenitors and suppressing osteoclast activity. Journal of Bone and Mineral Research, 12, 1797-1804. doi:10.1359/jbmr.1997.12.11.1797

[45] Venugopal, S.K., Devaraj, S., Yuhanna, I., Shaul, P. and Jialal, I. (2002) Demonstration that C-reactive protein decreases eNOS expression and bioactivity in human aortic endothelial cells. Circulation, 106, 1439-1441. doi:10.1161/01.CIR.0000033116.22237.F9

[46] Behl, Y., Krothapalli, P., Desta, T., Roy, S. and Graves, D. T. (2009) FOXO1 plays an important role in enhanced microvascular cell apoptosis and microvascular cell loss in type 1 and type 2 diabetic rats. Diabetes, 58, 917-925. doi:10.2337/db08-0537

[47] Venugopal, S.K., Devaraj, S. and Jialal, I. (2005) Effect of C-reactive protein on vascular cells: Evidence for a proinflammatory, proatherogenic role. Current Opinion in Nephrology and Hypertension, 14, 33-37. doi:10.1097/00041552-200501000-00006

[48] Alexiewicz, J.M., Kumar, D., Smogorzewski, M., Klin, M. and Massry, S.G. (1995) Polymorphonuclear leukocytes in non-insulin dependent diabetes mellitus: Abnormalities in metabolism and function. Annals of Internal Medicine, 123, 919-924.

[49] Grossi, S.G., Zambon, J.J., Ho, A.W., Koch, G., Dunford, R.G., Machtel, E.E., Norderyd, O.M. and Genco, R.J. (1994) Assessment of risk for periodontal disease. I. Risk indicators for attachment loss. Journal of Periodontology, 65, 260-267. doi:10.1902/jop.1994.65.3.260

[50] Sanchez, A.B.N., Almeida, R.F. and Martinez, A.B. (2007) Effects of non-surgical periodontal therapy on clinical and immunological response and glycemic control in type 2 diabetic patients with moderate periodontitis. Journal of Clinical Periodontology, 34, 835-843. doi:10.1111/j.1600-051X.2007.01127.X

[51] Lee, H.M., Golub, L.M., Chan, D., Leung, M., Schroeder, K., Wolff, M., Simon, M. and Crout, R. (1997) $\alpha$-proteinase inhibitor in gingival fluid of humans with adult periodontitis: Serpinloytic inhibition by doxycycline. Journal of Periodontal Research, 32, 9-19. doi:10.1111/j.1600-0765.1997.tb01377.x

[52] Hsu, M.J., Chang, C.K., Chen, M.C., Chen, B.C., Ma, H.P. and Hong, C.Y. (2010) Apoptosis signal-regulating kinase 1 in peptidoglycan induced COX-2 expression in macrophages. Journal of Leukocyte Biology, 87, 10691082. doi:10.1189/jlb.1009668

[53] Lalla, E., Kaplan, S., Yang, J., Roth, G.A., Papapanou, P.N. and Greenberg, S. (2007) Effect of periodontal therapy on serum C-reactive proteins, E-selectin, and tumour necrosis factor $\alpha$ secretion by peripheral blood-derived macrophages in diabetes. A pilot study. Journal of Periodontal Research, 42, 274-282. doi:10.1111/j.1600-0765.2006.00945.x 